IFN Working Paper No. 872, 2011

\title{
Income Inequality between Chinese Regions: Newfound Harmony or Continued Discord?
}

Johan Lyhagen and Johanna Rickne 


\title{
Income inequality between Chinese regions: Newfound harmony or continued discord?*
}

\author{
Johan Lyhagen \\ Department of Statistics, Uppsala University \\ Johanna Rickne ${ }^{\dagger}$ \\ Research Institute for Industrial Economics
}

May 9, 2011

\begin{abstract}
This paper develops an improved test of economic convergence or divergence using time series methods. The usefulness of the method is illustrated in an analysis of the growth pattern between Chinese regions in 1952-2007. Comparing all combinations of regional pairs, the analysis yields support for economic divergence in roughly half of the cases. In the other half, we instead find that regions have grown while maintaining stable income differences. As such, the results show the co-existence of divergence and conditional convergence among China's regions.

Keywords: China; Output convergence; Non-linear cointegration

JEL Classification: O53; R11; C32.
\end{abstract}

\section{Introduction}

After having sustained a $10 \%$ growth rate over the past 30 years, China is now the second largest economy in the world. The economic reform agenda that spurred this growth was highly diversified geographically. With the explicit goal of letting some provinces "get rich first", preferential policies aimed at first attracting technology and capital to localities along the coast. However, rather than the intended trickle down of growth to inland provinces, income inequalities between and within regions increased rapidly (Fan, Kanbur, and Zhang, 2009). Re-balancing these inequalities and achieving balanced, or "harmonious", growth continues to be a priority of the Chinese government.

A key prediction of the neoclassical growth model (Solow, 1956) is that of economic convergence, meaning that for given endowments, poorer economies

*The authors gratefully acknowledge helpful comments from Tor Eriksson, Bertil Holmlund, and Joakim Westerlund.

${ }^{\dagger}$ Financial support from the Marianne and Marcus Wallenberg Foundation is gratefully acknowledged. 
will eventually catch up with richer ones. This prediction has generated a large amount of empirical research on the existence of convergence between countries, and between regions within countries. In general, studies using cross-sectional methods have found evidence of conditional convergence (e.g. Baumol, 1986; Dorwick and Nguyen, 1989; Barro, 1991; Barro and Sala-i Martin, 1992; Mankiw, Romer, and Weil, 1992) while time series studies have concluded that economies do not converge (e.g. Bernard, 1992; Bernard and Durlauf, 1995; Quah, 1993).

One possible reason for the discrepancy in the empirical literature is that time series studies tend to use an inflexible test of convergence, requiring the difference between the two output series to be stationary with a zero mean ${ }^{1}$. But as noted by Bernard and Durlauf (1996), Harvey and Bates (2003) and Carvalho and Harvey (2005), this approach actually means testing whether convergence has already happened, which is completely different from the question of whether convergence is occurring.

In this paper we develop a new time series test that can determine whether income levels are in the process of becoming more similar or not, thus getting closer to testing the true convergence hypothesis. The test is constructed by introducing a nonlinear trend into the vector error correction model. By specifying this nonlinear trend as a logistic function we are able to estimate the trajectory of convergence or divergence by extracting the parameters associated with the estimated trend ${ }^{2}$. We can thus answer a number of hypotheses relating to the differences in growth pattern between China's regions ${ }^{3}$. In particular, we can plot the estimated trends for any cointegrating relationships between regions, and use the plots to determine graphically whether regions have: i) converged to the same income level (absolute convergence) or diverged to different income levels, ii) converged to different, but stable, income levels (conditional convergence), or iii) diverged and then converged, or vice-versa. In addition, we can analyze the timing, persistence, and changes between these situations over time.

The test is applied to Chinese regional data for the period $1952-2007^{4}$. We first consider the question of nationwide convergence, and then test for convergence within two classifications of regional subgroups. First, we divide regions into either Coastal or Central areas, the major political divide in the market reform period, during which the Coastal regions benefitted greatly from the Open Door policy. Next, we examine the existence of convergence among the regional sub-divisions of the North East, South West, North West and Central areas. Here, the North East represents the provinces that were the main locations of

\footnotetext{
${ }^{1}$ Following Proposition 5 of Bernard and Durlauf (1996), or equivalently in a panel setting as proposed by Evans (1998).

${ }^{2}$ A similar approach is the non-linear panel unit root test used by Lau (2010). There are some drawbacks with that method, e.g. the theory applied postulates that all differences between pairs of regions should cointegrate and that the test used only has the alternative hypothesis that a proportion of the differences is stationary.

${ }^{3}$ Following the literature, the term "region" is used to denote China's 22 provinces, 4 selfgoverning municipalities, and 5 Autonomous Regions. These three types of entities operate at the same administrative level and are directly subordinate to the central government.

${ }^{4}$ We also considered the reform period, 1978-2007, with very similar results to the ones presented for the longer period.
} 
the State-run heavy industries during the planned economy period, while the Central and South Western areas are cross-sections covering leading Coastal regions and their hinterlands. Finally, the North West area includes poor and landlocked interior regions.

The economic performances of China's 32 regions represent a suitable test bed for our model. Many of the regions are the same size as entire countries, but share institutions, language, and cultural traits which could cloud convergence tests in a cross-country setting. Previous empirical analyses of the convergence question also mirror the methodological divide mentioned above: cross-sectional studies have provided evidence in favor of economic convergence (Jian, Sachs, and Warner, 1996; Chen and Fleisher, 1996; Raiser, 1998; Weeks and Yao, 2003). In contrast, time series studies have documented economic divergence (Pedroni and Yao, 2006; Westerlund, Edgerton, and Opper, 2010). Using a more flexible convergence test has the potential for shedding light on the roots of this controversy.

Our paper does not simply contribute to the theoretical literature associated with time series tests of economic convergence; it also deepens our understanding of China's transition experience with respect to the critical question of income inequality. Our results show that divergence of economic output between roughly half of the region pairs started with market reform in the late 1970s, and showed no sign of reversal by 2007. Simultaneously, the other half of the region pairs grew at varying speeds while their income ratio remained relatively constant; this can be interpreted as indicating that conditional convergence was achieved.

The paper is organized as follows. Section 2 provides a literature review of studies of Chinese regional income convergence. Section 3 presents the data and Section 4 the model. Empirical results are contained in Section 5, and Section 6 outlines our conclusion.

\section{Literature review}

Early studies of economic convergence used cross sectional and panel data to produce regressions of regional growth rates on initial per capita income levels. These studies employed the term "unconditional convergence", also called $\beta$ convergence, to describe a negative coefficient on initial income (Barro and Sala-i Martin, 1997). In contrast, the concept of "conditional convergence" denotes the existence of a negative relationship after the inclusion of regional covariates that are expected to proxy for permanent differences in regional steady state income levels (Barro and Sala-i Martin, 1997).

In the Chinese case, cross-section and panel data studies that have used parametric regression methods have found evidence of economic catch-up of poor regions with richer ones. Jian, Sachs, and Warner (1996) did not find convergence in the pre-reform period, but showed that absolute convergence emerged strongly as a result of economic reform after 1978. Chen and Fleisher (1996), and Li, Zinan, and Rebelo (1998), found evidence to support both absolute and 
conditional convergence in the period 1978-1993. Cai, Wang, and Du (2002) and Raiser (1998) added support for conditional convergence as a consequence of market reform, and more recently, Zou and Zhou (2007) documented convergence within one group "developing" and one group of "developed" provinces, with faster convergence noted in the latter

Weeks and Yao (2003) note that the cross-sectional methodology to measure convergence can suffer from bias if the variables included fail to account for: i) unobserved province-specific heterogeneity in initial technology levels and ii) endogenous explanatory variables. Using Generalized Method of Moments estimation with province- and time-specific effects, they found that provinces were diverging in the pre-reform period, but that the interior and coastal regions converged to parallel underlying steady-states after 1979 .

Following criticism of the parametric regression methods used to investigate the convergence hypothesis (Quah, 1993, 1997; Maasoumi and Wang, 2007) ${ }^{5}$, researchers increasingly turned to time series analysis. Noting the common presence of unit roots in income per capita time series, Bernard and Durlauf (1991) and Quah (1993) proposed that stationarity of the difference between the income time series of two regions could be interpreted as convergence. The criterion was modified by Evans (1998) to fit panel data.

Pedroni and Yao (2006) employ Evan's method to investigate nationwide convergence between Chinese regions as well as convergence within various subgroups of regions in the pre-reform and reform periods. The authors construct a panel of relative income series by subtracting the average real income of the group under consideration (the cross-sectional mean) from the real income of each group member at each time point. Following Evans (1998), stationarity of the panel is interpreted as convergence within the group. Contrary to the results presented in earlier studies, the authors find evidence of income divergence, both nationally and within the vast majority of the examined subgroups. Lau (2010) extends the analysis of Pedroni and Yao by allowing for a non-linear unit root test, concluding that the main conclusions remain the same.

Westerlund, Edgerton, and Opper (2010) note that Evans' (1998) method, and the conclusions drawn by Pedroni and Yao (2006), may be sensitive to the choice of provinces included in each panel (and thereby in the calculation of the cross-sectional mean). Rather than relying on this calculation, they therefore conduct joint tests for unit roots in the income differences time series of all possible region pairs. The result, however, is no different: regions diverge economically, both nationally and in various sub-samples (Westerlund, Edgerton, and Opper, 2010).

\footnotetext{
${ }^{5}$ Evans and Karras (1996) argue that the conventional cross-sectional technique of testing for economic convergence produces invalid inferences unless all permanent cross-economy differences in per capita GDP are perfectly controlled for and unless the countries or regions under consideration have income series that exhibit identical first-order autoregressive dynamic structures.
} 


\section{Data}

The Hsueh and Li (1999) GDP data for Chinese regions in the period 19521995 are combined with data from China's Statistical Yearbook for 1996-2007. ${ }^{6}$ Regional GDP per capita series at 1995 prices are calculated using information on total regional population and regional GDP deflators from China's statistical yearbook. As in previous studies, we exclude the regions of Hainan and Tibet, the two smallest regional economies, for which data are missing prior to 1987 and 1988, respectively. To allow a comparison over time, Chongqing is treated as part of the Sichuan region from which it was separated in 1997. Information on the geographical classification of our regions is presented in Table 1. We use two categorizations: the division "Interior" vs. "Coastal", and the division between "Central", "North East", "North West", and "South West" areas.

Figures 1-3 provide a graphical overview of the general trends with respect to income convergence. Using first the classification of regions as belonging to either the Interior (10 regions) or the Coastal area (18 regions), Figure 1 shows the mean income level per capita of the two areas relative to the national average. We see that the average Coastal region developed faster than the average Interior region, and that the income gap between these two areas widened during the reform period. Figures 2 and 3 illustrate the within-group heterogeneity by plotting the growth paths of the poorest and wealthiest two regions within the Coastal and Interior areas, respectively. Figure 2 shows that the richest Coastal province (Shanghai) grew at a roughly similar pace to the poorest Coastal region (Hebei). Figure 3 shows a similar pattern for the regions located in the Interior. During the sample period, the log of real GDP per capita in the poorest Interior region (Guizhou) remained at an apparently constant ratio to the wealthiest region (Beijing).

\section{Model}

This section describes the econometric method used in the paper. The data are transformed using natural logarithms. As there is a rather large number of regions, i.e. 28 , it is not possible to conduct a full system/multivariate analysis.

As is well known, see references above, differences between pairs of regions or between a region and the average of all regions should be stationary although the GDP for each region is a unit root process. As pointed out by Westerlund, Edgerton, and Opper (2010), there are some problems with the analysis of pairs of regions and the average of the regions. The unit root approach implies that the cointegrating vector should be $[1,-1]$ between each pair of regions. This is not necessarily true and the unit root test would, in this case, not reject the null hypothesis of a unit root.

Our starting point is the (bivariate) vector autoregressive model, VAR. The VAR, in levels, is used to choose lag lengths for each pair of regions based on

\footnotetext{
${ }^{6}$ Following Pedroni and Yao (2006); Lau (2010) and Westerlund, Edgerton, and Opper (2010).
} 


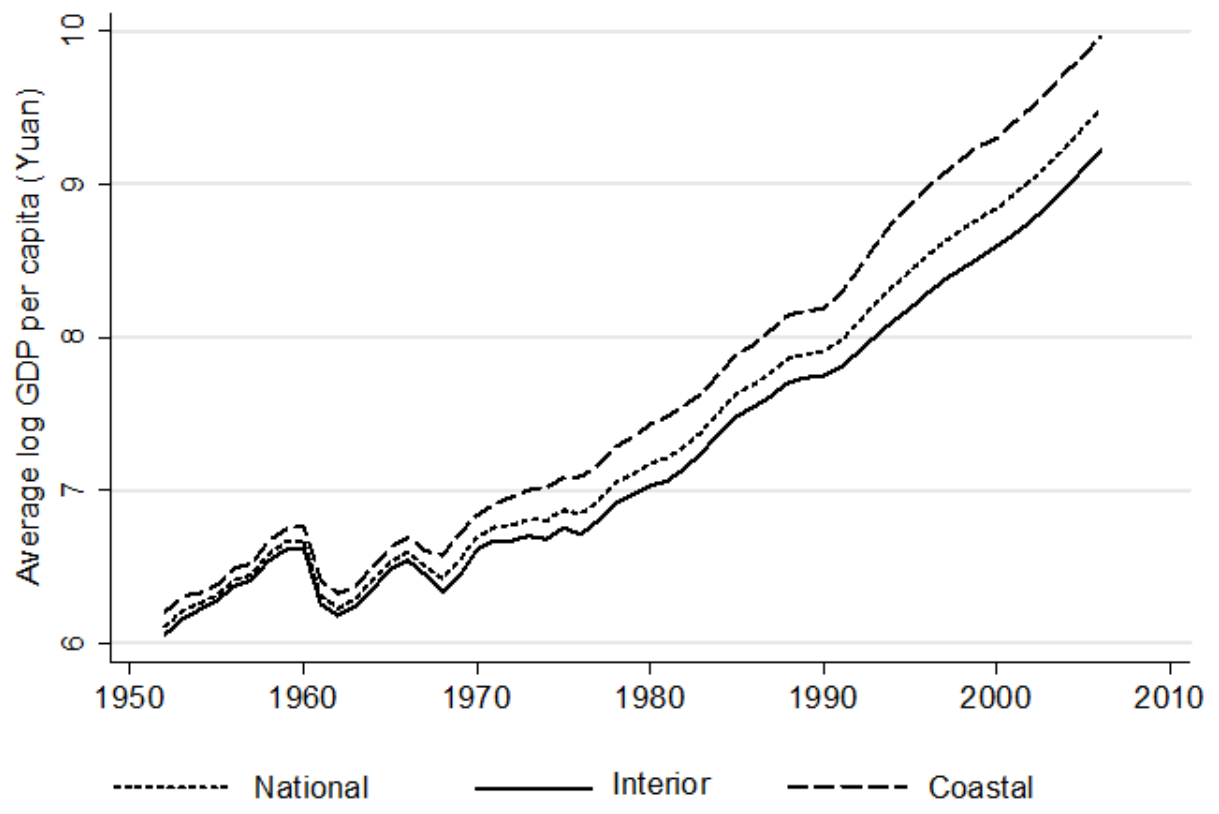

Figure 1: Average log GDP per capita for the full sample (National), and the Interior and Coastal areas, 1952-2007. 


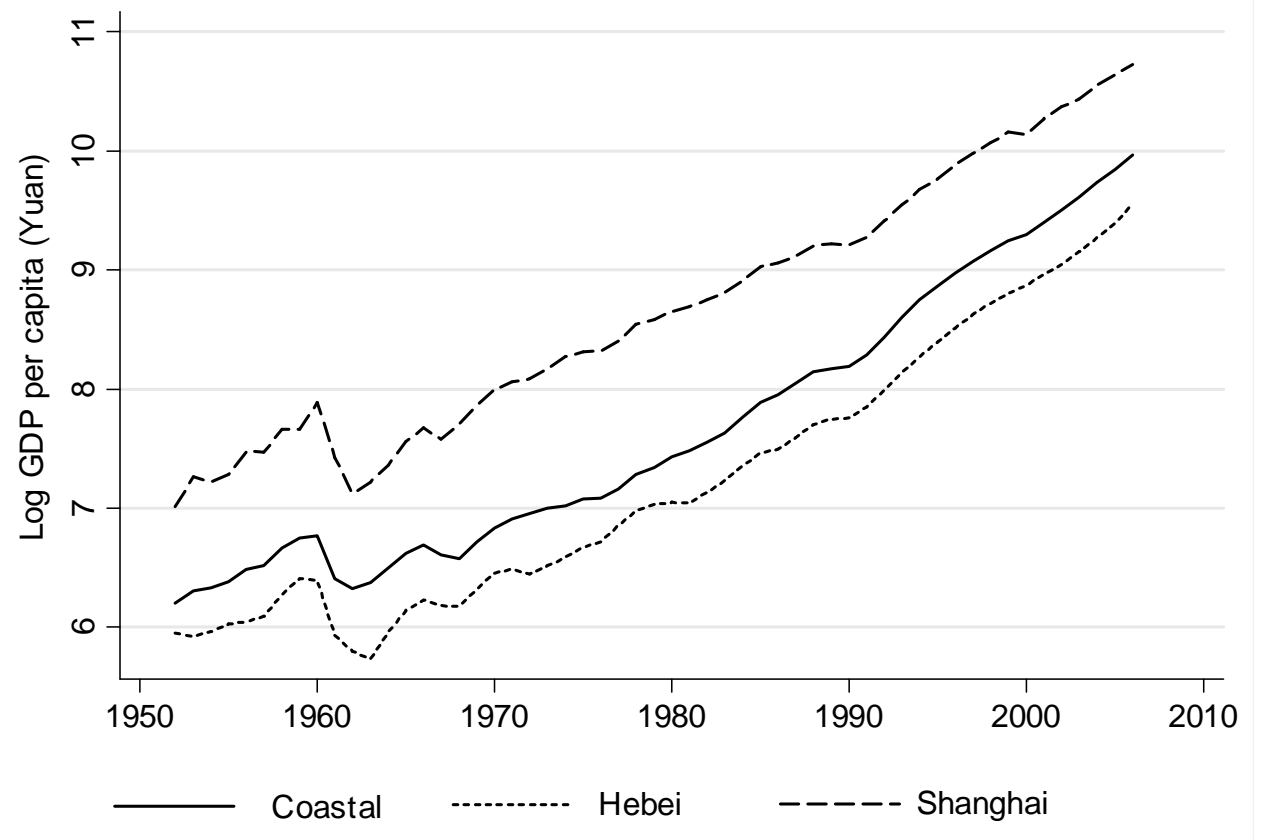

Figure 2: Growth paths for the poorest and richest Coastal regions relative to the Coastal average, 1952-2007. 


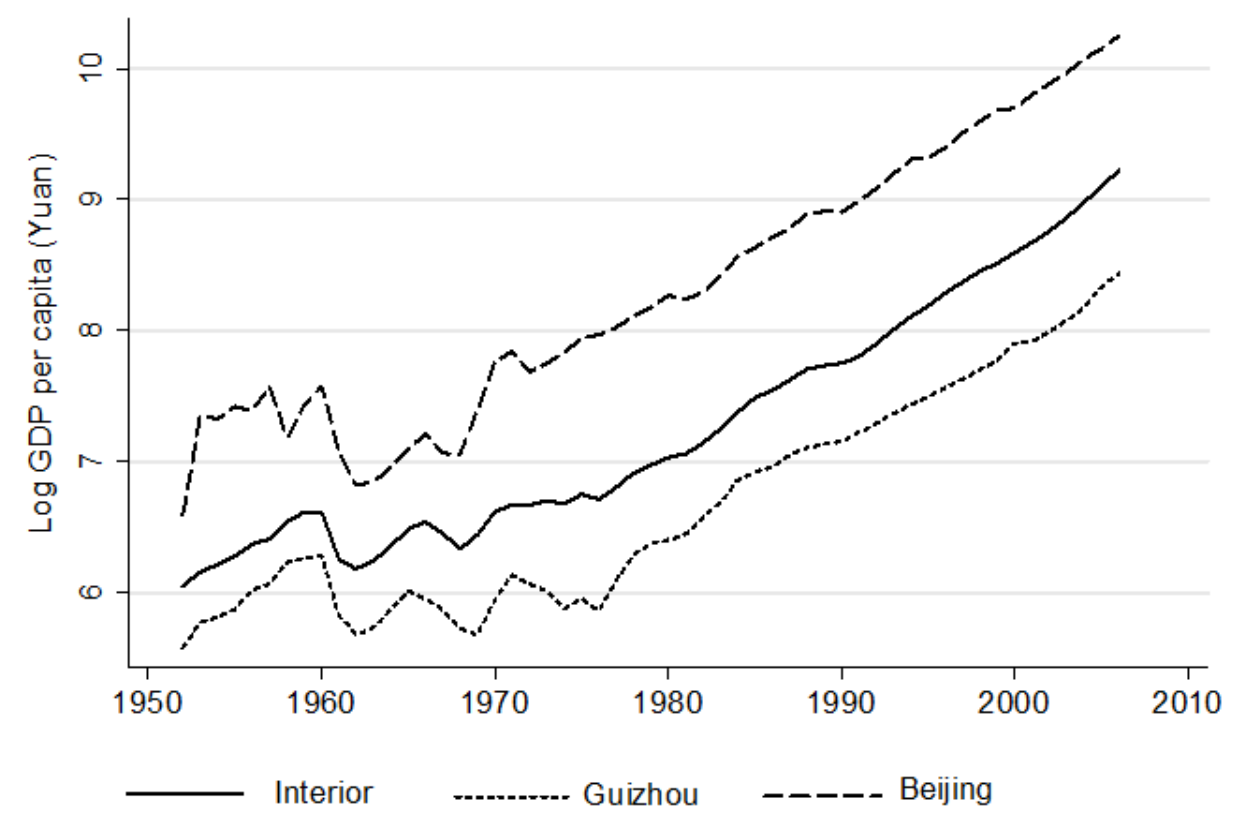

Figure 3: Growth paths for the poorest and richest Interior regions relative to the Interior average, 1952-2007. 
Table 1: Classification of regions into geographical areas

\begin{tabular}{lll}
\hline Region & Classification 1 & Classification 2 \\
\hline Beijing & Interior & Central \\
Tianjing & Coastal & Central \\
Hebei & Coastal & Central \\
Shanxi & Interior & Central \\
Mongolia & Interior & NW \\
Liaoning & Coastal & NE \\
Jilin & Interior & NE \\
Heilongjiang & Interior & NE \\
Shanghai & Coastal & Central \\
Jiangsu & Coastal & Central \\
Zhejiang & Coastal & Central \\
Anhui & Interior & Central \\
Fujian & Coastal & Central \\
Jiangxi & Interior & Central \\
Shandong & Coastal & Central \\
Henan & Interior & Central \\
Hubei & Interior & Central \\
Hunan & Interior & Central \\
Guangdong & Coastal & Central \\
Guangxi & Coastal & SW \\
Sichuan & Interior & SW \\
Guizhou & Interior & SW \\
Yunnan & Interior & SW \\
Shaanxi & Interior & NW \\
Gansu & Interior & NW \\
Qinghai & Interior & NW \\
Ningxia & Interior & NW \\
Xinjiang & Interior & NW \\
\hline
\end{tabular}


the Schwarz information criterion. The next step is to perform the Johansen likelihood ratio test for the cointegrating rank. Because the growth of regions is interdependent, we conduct residual bootstrapping, in which the data are generated for the pairs using residuals from the same point in time. It is well known that the bootstrap method works considerably better than using asymptotic critical values, see e.g. Swensen (2006).

The problem with the cointegration approach described above is that it is only valid if convergence has already taken place, and not when convergence is occurring, as previously noted by Harvey and Bates (2003) and Carvalho and Harvey (2005). To deal with this we introduce a deterministic variable to capture this phenomenon. This variable is a logistic function ${ }^{7}$ :

$$
g_{t}=\frac{1}{1+e^{-\gamma(t-\delta)}}
$$

The VECM becomes:

$$
\Delta X_{t}=\mu+\alpha\left(\begin{array}{ll}
\beta^{\prime} & \rho^{\prime}
\end{array}\right)\left(\begin{array}{c}
X_{t-1} \\
g_{t}
\end{array}\right)+\sum_{i=1}^{k-1} \Gamma_{i} \Delta X_{t-i}+\varepsilon_{t}
$$

where $\mu$ is an unrestricted constant, $\varepsilon_{t}$ is a random vector with covariance matrix $\Omega$ etc., see Johansen (1995). It is possible to use this model to test for the number of cointegrating vectors; the asymptotic distribution of the likelihood ratio test is given by Johansen and Nielsen (1993). In addition, in this case, we bootstrap to obtain an approximation of the small sample distribution. Furthermore, it is possible to test the null hypothesis that $\rho=0$ using the likelihood ratio test, although the distribution is non-trivial due to the non-identifiability of $\gamma$ and $\delta$ under the nullhypothesis. The bootstrap approach is also used here. To determine whether there was first a period of divergence, and then a period of convergence (or vice-versa), a second logistic function is added and the tests for this situation followed those outlined above.

The estimation of the non-linear VECM is non-trivial as it is non-linear with respect to the parameters, but, depending on $\gamma$ and $\delta$, the model is linear for the remaining parameters. Hence, given $\gamma$ and $\delta$ it is trivial to calculate the likelihood and we use a numerical optimization routine to optimize $\gamma$ and $\delta$. This is performed using MATLAB version 2009a.

\section{Empirical results}

\subsection{Cointegration tests}

Table 2 presents the test results for the cointegrating rank of each geographical area under consideration, as well as tests of non-linearity in the trends of the cointegrating relationships. Column 1 presents the p-values for the standard

\footnotetext{
${ }^{7}$ Due to identification of the parameters, without loss of generality, the first observation is normalized to zero and the last to one.
} 
Johansen test for no cointegration, $r=0$, when including a linear trend and a constant in the cointegrating relationship. The null hypothesis is rejected for all samples except the North Eastern and South Western sub-groups. These rejections can be interpreted in two ways. On one hand, it is more difficult to reject the null hypothesis of no cointegration when we have fewer observations. On the other hand, the test may erroneously show no cointegration if the cointegrating relationship has a non-linear rather than a linear trend.

Next, we conduct the Johansen test for the null of no cointegration $r=0$ versus of a cointegrating relationship with either 1) a nonlinear trend with one nonlinear element (Column 2) or 2) with two nonlinear elements (Column 3). The results clearly indicate that we should reject the null hypothesis for all subsamples in the cases of both trend specifications. Thus, the results support the previous conjecture regarding the erroneous acceptance of the null hypothesis (i.e. no cointegration) in Column 1 due to incorrect specification of the trend. We could however still have a situation of no cointegration if the rank is two rather than one, in which case both time series constitute separate stationary processes. Testing for this property (Column 4) does not lead us to reject the null hypothesis of one cointegrating relationship for any of the samples.

Having reached the conclusion that there is one cointegrating relationship, we next investigate the statistical significance of the nonlinear trend parameters. The results presented in Columns 5-6 relate to applying the likelihood ratio test to a single non-linear element (Column 5), or to two nonlinear elements (Column 6 ). The p-values indicate strong support for the statistical significance of the trend parameters, regardless of the test. ${ }^{8}$

We still need to exclude the possibility that there are two stationary processes (no cointegration) and nonlinear trends. Column 7, therefore, shows the result of testing the hypothesis that there is one cointegrating relationship $(r=1)$ against the alternative that there are two cointegrating relationships $(r=2)$ with nonlinear trends. In this case, we consistently accept the hypothesis that there is one cointegrating relationship.

The last step of the analysis is to determine the precise nonlinear characteristics of the trends. The final column in Table 2 shows the results of comparing our two trend specifications, where the null hypothesis is specified as the existence of one nonlinear element. The p-values indicate that we should accept the null hypothesis in half of the cases. It is accepted for the Coastal and the Central areas. For the Interior area, the North West, the North East, and the South West we instead find evidence of double nonlinearity.

\subsection{Graphical analysis of nonlinear cointegrating relation- ships}

It is important to remember that providing empirical evidence for the existence of a nonlinear trend in the cointegrating relationship between the real GDP per

\footnotetext{
${ }^{8} \mathrm{~A}$ test based on the Taylor approximation principle presented by Teräsvirta (1994) was also carried out. The result was in accordance with the other tests, hence not reported.
} 
Table 2: P-values for the hypotheses relating to cointegrating rank and functional form of the deterministic term for various groups, 1952-2007.

\begin{tabular}{lcccccccc}
\hline Hypothesis/group & 1 & 2 & 3 & 4 & 5 & 6 & 7 & 8 \\
\hline All & 0.008 & 0.000 & 0.000 & 0.120 & 0.000 & 0.000 & 1.000 & 0.000 \\
Coastal & 0.055 & 0.000 & 0.000 & 0.070 & 0.000 & 0.000 & 1.000 & 0.000 \\
Interior & 0.003 & 0.000 & 0.000 & 0.170 & 0.000 & 0.000 & 1.000 & 0.261 \\
Central & 0.025 & 0.000 & 0.000 & 0.138 & 0.000 & 0.000 & 1.000 & 0.000 \\
NW & 0.040 & 0.000 & 0.000 & 0.411 & 0.000 & 0.000 & 1.000 & 0.985 \\
NE & 0.160 & 0.008 & 0.038 & 0.218 & 0.000 & 0.003 & 1.000 & 1.000 \\
SW & 0.306 & 0.003 & 0.045 & 0.175 & 0.000 & 0.000 & 1.000 & 0.223 \\
\hline
\end{tabular}

Notes: The hypotheses were as follows, where the type of deterministic term is restricted to the long run relationship and $r$ is the cointegrating rank: 1) constant, $\left.H_{0}: r=0, H_{A}: r=2 ; 2\right)$ constant and logistic function, $H_{0}: r=0$, $H_{A}: r=2$; 3) constant and two logistic functions, $H_{0}: r=0, H_{A}: r=2$; 4) constant $\left.H_{0}: r \leq 1, H_{A}: r=2 ; 5\right)$ constant and $r=1, H_{0}$ :linear, $H_{A}$ : two logistic functions; 6 ) constant and $r=1, H_{0}::$ linear, $H_{A}$ : two logistic functions; 7) constant and logistic function $r=1, H_{A}: r=2$, 8) $r=1, H_{0}$ : one logistic function, $H_{A}$ : two logistic functions.

capita series of two regions does not mean that we have evidence of convergence. If the trend is upwards, this means that the regions are in fact diverging. Only a negative trend shows that the income levels of two regions are becoming more similar.

The main conclusions about convergence or divergence between China's regions are drawn by examining graphical plots of the cointegrating relationships between the regional pairs. By studying these plots, we are able to address three key issues. First, we want to examine the slope of the nonlinear trends to determine whether the region pairs experienced divergence (upward slope) or convergence (downward slope). Second, we want to evaluate the magnitude of the gradients of the slopes. Steep lines indicate rapid processes of convergence or divergence. Straight lines indicate that the region pairs have grown while keeping a constant income gap. Thus, we can interpret straight lines as conditional convergence in the case of a nonzero mean, and unconditional convergence in the case of a zero mean. Third, we want to inspect visually the distribution of trends in each group of regions to determine the relative occurrence of convergence or divergence.

Using the information in Column 8 of Table 2 to guide the choice of trend specification, we plot region pair trends using either single or double nonlinearity. Because of the large number of region pairs, for each given year the trends are first ranked by size, and the distribution of trends then illustrated by quantiles. Looking at Figure 4, which illustrates the distribution of region-pair trends for the nationwide sample, we can interpret the lines in the figures as follows. The middle of the five indicator lines shows the median trend, meaning that $50 \%$ of all the region-pair trends lie above this line. The second indicator 
line from the top delineates the top $25 \%$ of the trends, and $0.025 \%$ of the trends are above the uppermost line. Analogously, the two bottom lines indicate the lowest $25 \%$ and $0.025 \%$ of the trends.

In Figure 4, the distribution of trends shows a clear pattern of upward sloping nonlinear trends for more than $50 \%$ of the regional pairs. Focusing on the year indicators on the X-axis, we can also observe that this divergence, as indicated by the upward sloping trends, started during the 1970s, but accelerated for some region pairs from the early 1980s. Zooming in on the right side of the graph, there are no directional changes in the lower four lines, but a small kink in the uppermost line in the mid 90s. These observations demonstrate that the process of divergence has largely not lost pace in recent years. The exception is the most rapidly diverging region pairs, where the process slowed down slightly from the mid 90s and onward.

For the remaining and non-diverging pairs, we can see flat trends, most with nonzero means. Thus, the results do not support ongoing convergence, but rather that the regions were growing along separate but different trajectories, retaining constant income differences rather than becoming more similar.

We next dissect the nationwide pattern and examine smaller groups of regions to see whether we can identify areas of divergence and conditional convergence. Figure 5 shows the trend distributions of the region pairs in the Interior and the Coastal areas, respectively. Comparing the two figures, we note that the trend distributions appear very similar, with a mixed picture of divergence and convergence between the various region pairs. Upward trends show that divergence has occurred between more than $50 \%$ of the pairs in each of the two areas. However, there are also slightly steeper gradients, that is, faster divergence, for the most rapidly diverging pairs in the interior area. With regards to the timing of divergence, the coastal regions appear to have grown apart more consistently over the entire $1952-2007$ period. In contrast, we note that divergence in the interior regions appears to be more directly associated with the economic reforms of the past three decades.

Comparing the regions in the lower part of Figure 5, we see that many of the flatter trends for the cointegrating relationships among the Coastal regions are associated with means that are closer to zero. This implies that the pairs of Coastal regions that have grown at very similar rates have had more equal income levels, while there are larger and persistent income differences among the regions in the Interior part of the country.

Smaller subdivisions of the region pairs are presented in Figure 6, which shows the distribution of trends for the region pairs within the North East and South Western groups, and Figure 7, which shows the trends for the region pairs in the Central and North Western regions.

In the North Eastern provinces of Heilongjiang, Jilin and Liaoning the figure indicates that the income levels for these regions have not converged. Rather, the pace of divergence accelerated in the post-1979 period, indicating that the dramatic privatization and retrenchment of state-owned industries was conducive to increased growth dispersion between these three regions. In the South Western regions of Guangxi, Sichuan, Giuzhou and Yunnan the growth pattern ap- 


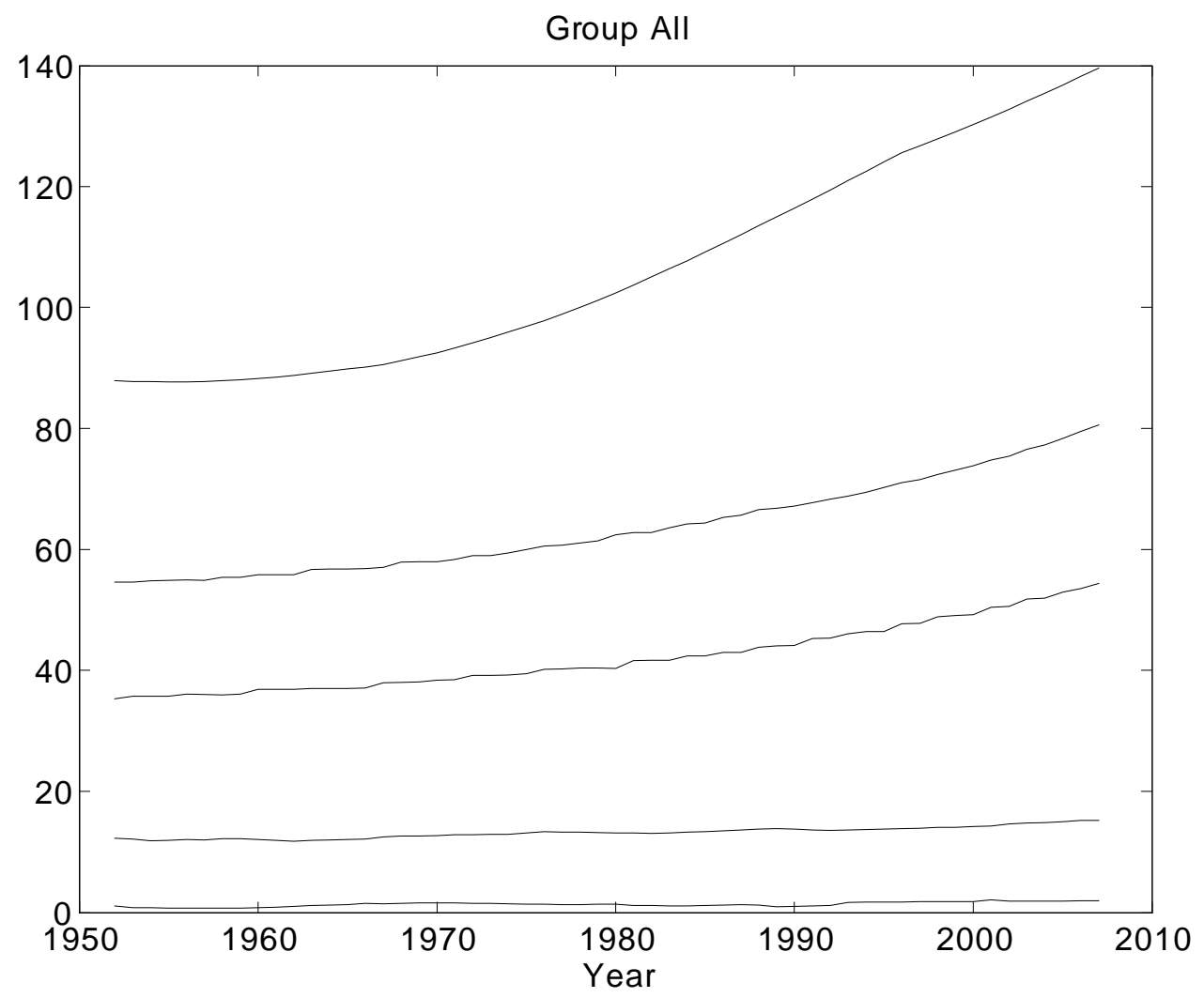

Figure 4: Quantiles $0.025,0.25,0.5,0.75$, and 0.975 of the absolute value of the long run deterministic terms for all region pairs.

pears more stable than for the Northern coast. Only one province pair diverged markedly in the post-1979 period.

Finally, we consider the Central and North Western areas. Because of the larger number of regions in the Central sub-division, the figure shows the trend distribution rather than the individual region pair trends as for the North West. In these regions, the growth pattern in both areas mirrors the nationwide results. While about half of the regions have followed diverging growth trajectories, there is also a substantial proportion of regions which have grown at different paces while keeping one regions income per capita as a constant ratio of the other region's income per capita. This evidence may be interpreted as the regions having achieved conditional convergence. In some cases, the difference in income has been very small, as shown by the trend lines close to zero, indicating that a few regions experienced quite similar income levels during the entire period under investigation. 

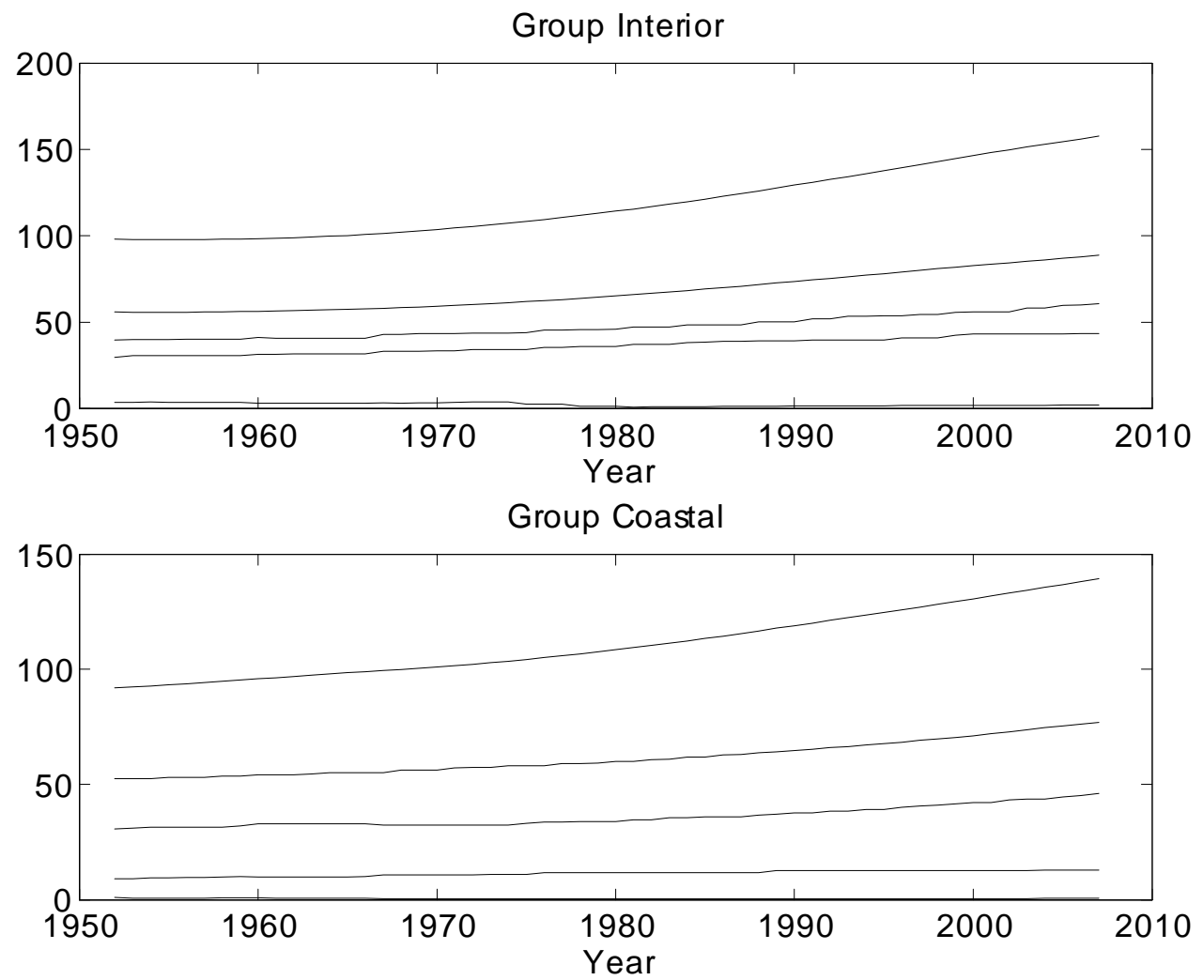

Figure 5: Quantiles 0.025, 0.25, 0.5, 0.75, and 0.975 of the absolute value of the long run deterministic terms for all region pairs in the Coastal and Interior geographical groups. 

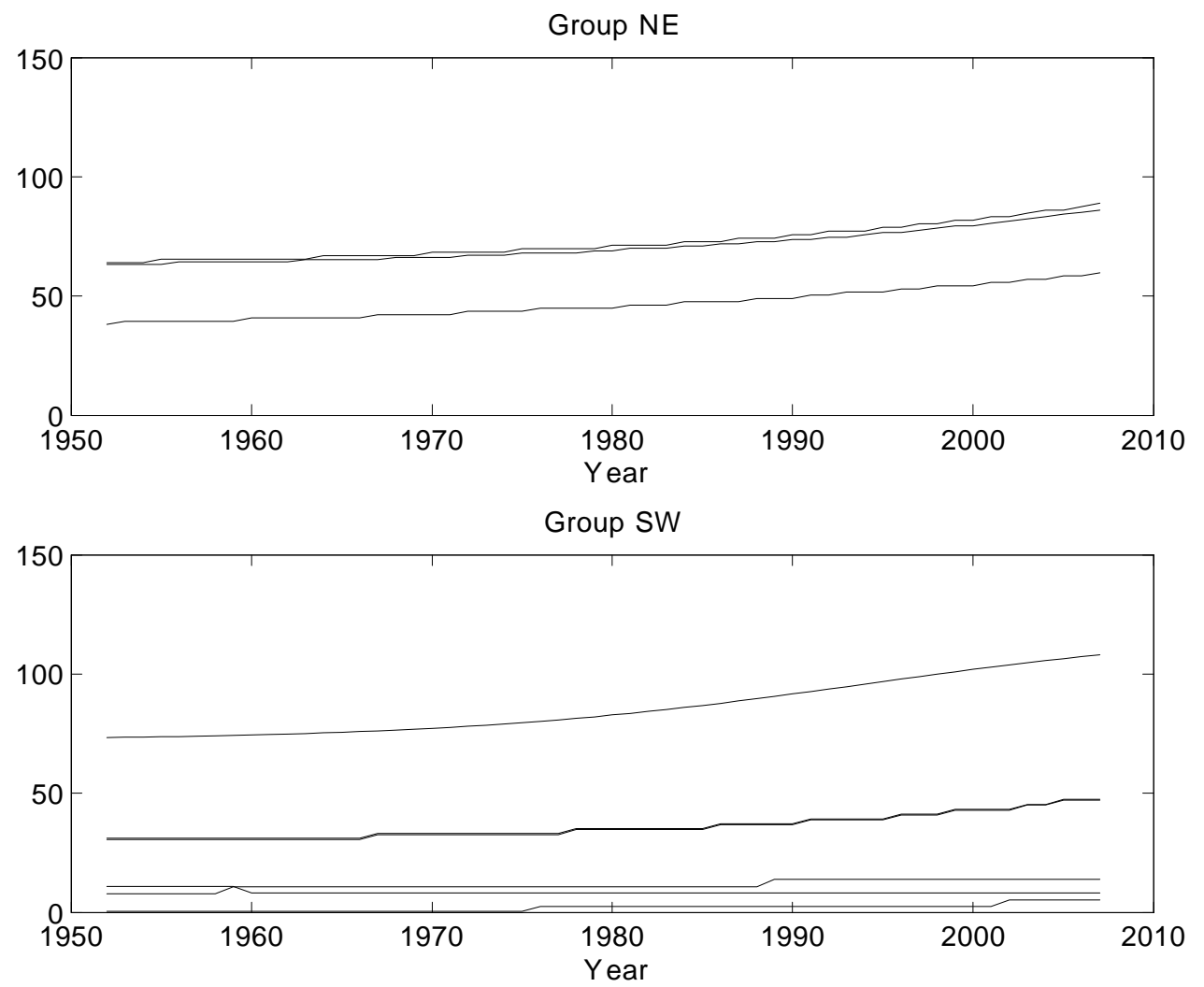

Figure 6: Absolute values of the individual deterministic trends for the North East and South West groups. 

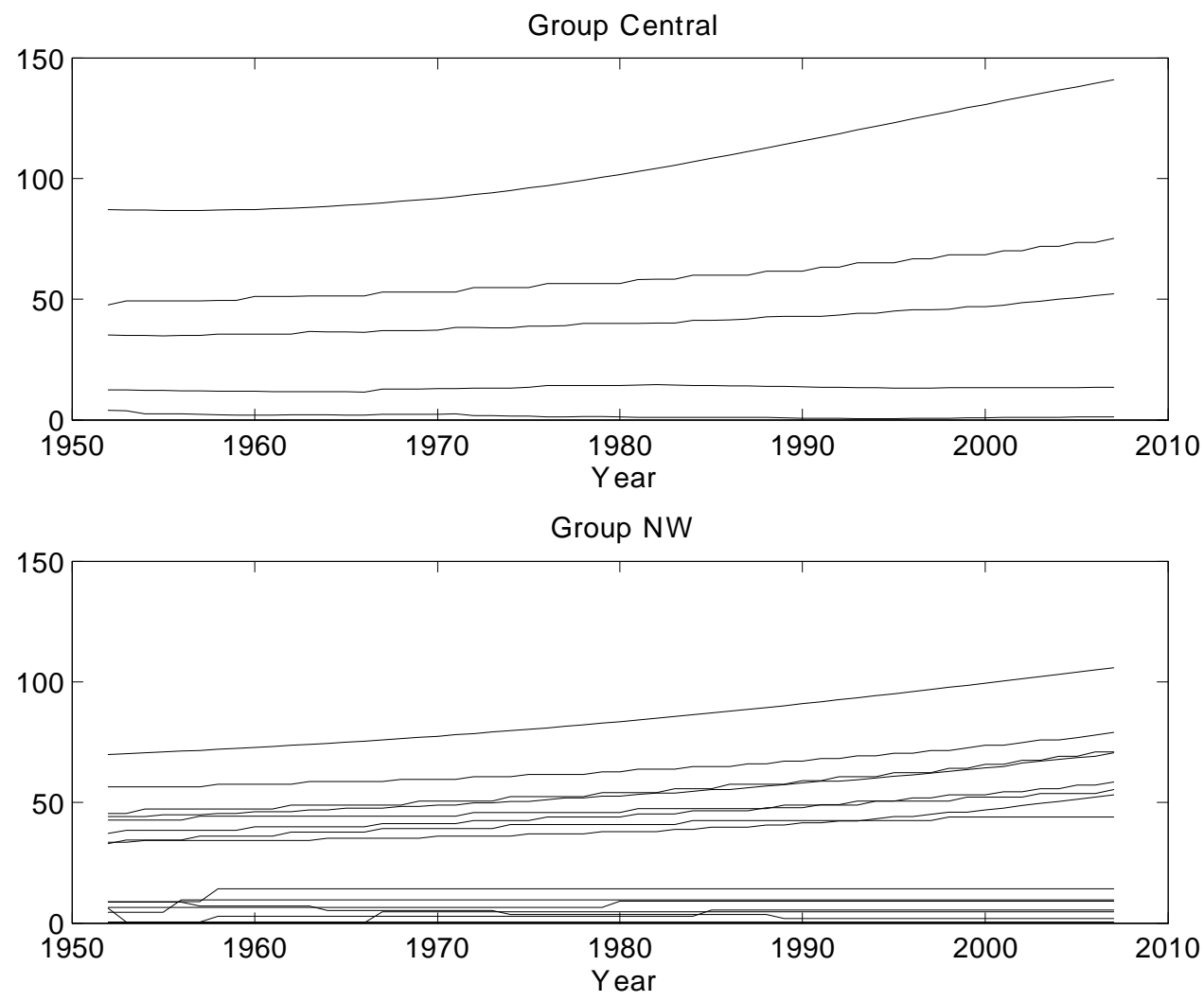

Figure 7: Quantiles 0.025, 0.25, 0.5, 0.75, and 0.975 of the absolute value of the long run deterministic terms for all region pairs in the Central geographical group, and the absolute value of the individual deterministic trends for the North Western group. 


\section{Conclusions}

In this paper we presented a new method to test for economic convergence or divergence. Using Chinese regions as a case study we found that out test specification performed well compared to the previously suggested methods (e.g. Evans, 1998). Cointegration tests within the VAR model applied to the output differences between all possible regional pairs 1 ed us to reject the hypothesis of cointegrating relationships with linear trends in favor of the occurrence of nonlinear trends. Thus, we found that our test specification captured more of the growth dynamics between regions than previous methods. The improved model fit leads us to conclude that our approach is more likely to produce correct answers to the convergence question.

Compared to previous time series studies of convergence in China, our method allowed a flexible assessment of convergence and divergence nationwide and within groups of regions, rather than the need to conclude in favor of either one pattern or the other (e.g. Pedroni and Yao, 2006; Westerlund, Edgerton, and Opper, 2010). Examining the trends of the cointegrating relationships between regional pairs, we found that although divergence was taking place among approximately half of the pairs, the other half neither diverged nor converged during the period 1952-2007. Instead, the income ratio between these regions remained roughly constant, suggesting that they had achieved conditional convergence.

A closer graphical examination of the trends associated with the cointegrating relationships for the region pairs also yielded a number of insights. Unlike previous time series studies, we were able to show - without reducing the number of observations by dividing the data into pre- and post-reform samples that economic divergence accelerated in the reform period. By allowing for both single and double nonlinearities, and then analyzing the direction and inclination of the estimated trends, we were able to reject the hypothesis that regions had diverged at the beginning of the reform period and then converged in more recent years. Despite the finding of a slight slowdown in the divergence between the fastest diverging provinces since the mid 1990s, we found little evidence of success for the last decade's investment efforts aimed at bridging the economic gap between the East and West by boosting the development of the Western regions.

\section{References}

BARRO, R. J. (1991): "Economic growth in a cross section of countries," Quarterly Journal of Economics, 106(2), 385-406.

Barro, R. J., and X. Sala-I Martin (1992): "Convergence," The Journal of Political Economy, 100(2), 223-251.

(1997): "Technological Diffusion, Convergence and Growth," Quarterly

Journal of Economics, 2(1), 1-26. 
BAumol, W. W. (1986): "Productivity growth, convergence, and welfare: What the long-run data show," American Economic Review, 76(5), 10721085.

Bernard, A. B. (1992): "Empirical implications of the convergence hypothesis," Discussion paper, Working Paper MIT Cambridge, MA.

Bernard, A. B., and S. N. Durlauf (1991): "Convergence in international output movements," Discussion paper, NBER Working Paper No. 3717.

(1995): "Convergence in international output," Journal of Applied Econometrics, 10(2), 97-108.

(1996): "Interpretating tests of the convergence hypothesis," Journal of Econometrics, 71(1-2), 161-171.

CaI, F., D. Wang, and Y. Du (2002): "Regional disparity and economic growth in China: The impact of labor market distortions," China Economic Review, 13(2-3), 197-212.

Carvalho, V., and A. C. Harvey (2005): "Growth, cycles and convergence in US regional time series," International Journal of Forecasting, 21(4), 667686.

Chen, J., and B. M. Fleisher (1996): "Regional income inequality and economic growth in China," Journal of Comparative Economics, 22(2), 141-164.

Dorwick, S., And D.-T. NGuyen (1989): "OECD Comparative Economic Growth 1950-85: Catch-Up and Convergence," American Economic Review, 79(5), 1010-1030.

Evans, P. (1998): "Using Panel Data to Evaluate Growth Theories," International Economic Review, 39(2), 249-265.

Evans, P., And G. KarRas (1996): "Convergence revisited," Journal of Monetary Economics, 37(2), 249-265.

Fan, S., R. Kanbur, and X. Zhang (2009): Regional Inequality in China: Trends, Explanations and Policy Responses. Routledge UK.

Harvey, A., and D. Bates (2003): "Multivariate Unit Root Tests and Testing for Convergence," Discussion paper, Cambridge Working Paper in Economics No. 0301.

Hsuen, T.-T., And Q. Li (1999): China's National Income 1952-1995. Westview Press, Oxford.

Jian, T., J. D. Sachs, and A. M. Warner (1996): "Trends in regional inequality in china," China Economic Review, 7(1), 1-21. 
Johansen, S. (1995): Likelihood-Based Inference in Cointegrated Vector Autoregressive Models. Oxford University Press, Oxford.

Johansen, S., And B. Nielsen (1993): "Manual for the simulation program DisCo," Institute of Mathematical Statistics, University of Copenhagen.

LAU, C. K. M. (2010): "New evidence about regional income divergence in China," China Economic Review, 21(2), 293-309.

Li, H., L. Zinan, and I. Rebelo (1998): "Testing the neoclassical theory of economic growth: Evidence from Chinese provinces," Economic Planning, 31(2-3), 117-132.

MaAsoumi, E., and L. Wang (2007): "Economic Reform, Growth and Convergence in China," Econometrics Journal, 10, 1-25.

Mankiw, G. N., D. Romer, and D. N. Weil (1992): "A Contribution to the Empirics of Economic Growth," Quarterly Journal of Economics, 107(2), 407-437.

Pedroni, P., and J. Yao (2006): "Regional income divergence in China," Journal of Asian Economics, 17, 294-315.

QuAH, D. T. (1993): "Galton's fallacy and tests of the convergence hypothesis," Scandinavian Journal of Economics, 95(4), 427-443.

(1997): "Empirics for growth and distribution: polarization, stratification and convergence clubs," Journal of Economic Growth, 2, 127-59.

RaISER, M. (1998): "Subsidising Inequality: Economic Reforms, Fiscal Transfers and Convergence across Chinese Provinces," Discussion paper, Kiel Working Paper No. 758.

Solow, R. (1956): "A Contribution to the Theory of Economic Growth," Quarterly Journal of Economics, 70(1), 65-94.

Swensen, A. (2006): "Bootstrap Algorithms for Testing and Determining the Cointegration Rank in VAR Models," Econometrica, 74, 1699-1714.

TERÄSvirTA, T. (1994): "Specification, estimation and evaluation of smooth transition autoregressive models," Journal of the American Statistical Association, 89(425), 208-218.

Weeks, M., and J. Y. Yao (2003): "Provincial conditional income convergence in china, 1953-1997," Economic Reviews, 22(1), 59-77.

Westerlund, J., D. L. Edgerton, and S. Opper (2010): "Why is Chinese provincial output diverging?," Journal of Asian Economics, 21(4), 333-344.

Zou, W., and H. Zhou (2007): "Classification of Growth Clubs and Convergence: Evidence from Panel Data in China, 1981-2004," China 83 World Economy, 15(5), 91-106. 